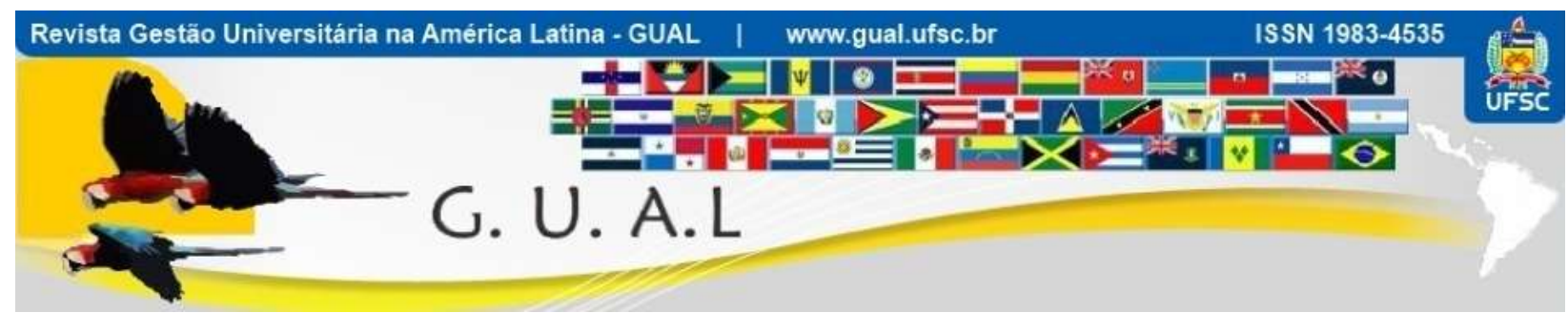

DOI: http://dx.doi.org/10.5007/1983-4535.2020v13n2p183

\title{
ALINHAMENTO ESTRATÉGICO DE PLANEJAMENTOS INSTITUCIONAIS: ESTUDO EM UMA UNIVERSIDADE FEDERAL DA AMAZÔNIA SETENTRIONAL
}

\section{STRATEGIC ALIGNMENT OF INSTITUTIONAL PLANNING: STUDY AT A FEDERAL UNIVERSITY IN THE NORTHERN AMAZON}

\author{
Ananias Costa Oliveira, Mestre \\ https://orcid.org/0000-0001-7039-3768 \\ ananias.staff@gmail.com \\ Universidade Federal do Amapá | Divisão de Acompanhamento e Avaliação \\ Macapá | Amapá | Brasil \\ Francisco Roberto Pinto, Doutor \\ https://orcid.org/0000-0003-2559-1524 \\ roberto.pinto@uece.br \\ Universidade Estadual do Ceara | Programa de Pós-Graduação em Administração \\ Fortaleza | Ceará| Brasil \\ Cláudio Márcio Campos de Mendonça, Doutor \\ http://orcid.org/0000-0001-7493-3469 \\ cmarcio@gmail.com \\ Macapá | Amapá | Brasil
} Universidade Federal do Amapá | Mestrado Profissional em Propriedade Intelectual e Transferência de Tecnologia

Recebido em 04/setembro/2019

Aprovado em 29/janeiro/2020

Publicado em 01/maio/2020

Sistema de Avaliação: Double Blind Review 


\title{
RESUMO
}

O objetivo deste estudo incidiu em identificar o alinhamento entre o plano de desenvolvimento institucional e o planejamento estratégico de gestão de pessoas da Universidade Federal do Amapá. O desenho metodológico consistiu em uma pesquisa exploratória e explicativa-descritiva, de abordagem predominantemente qualitativa, cujos dados coletados advieram de legislações governamentais, planejamentos institucionais e de entrevistas com gestores e servidores. Na etapa analítica, utilizou-se a técnica análise de conteúdos, com auxilio do software ATLAS.ti 8 - Qualitative Data Analysis. Os resultados identificaram alinhamento entre o PDI (2015-2019) da UNIFAP e o Planejamento Estratégico da PROGEP, especialmente no uso de métodos e ferramentas similares no processo de formulação estratégica, como Mapas Estratégicos e Balanced Scorecards. Os mapas estratégicos demonstraram encadeamentos entre objetivos estratégicos globais e setoriais, para o desdobramento da filosofia institucional para o campo da gestão de pessoas.

Palavras-chave: Administração Pública. Consistência Estratégica. Alinhamento Institucional.

\begin{abstract}
The objective of this study was to identify the alignment between the institutional development's plan and the strategic planning of people management at Universidade Federal do Amapá (Federal University of Amapá). The methodological design consisted of an exploratory and explicative-descriptive research, with a predominantly qualitative approach, whose collected data came from governmental legislation, institutional planning and interviews with managers and servers. In the analytical stage, the technique of content analysis was used, with the help of ATLAS.ti 8 - Qualitative Data Analysis software. The results identified alignment between the UNIFAP IDP (2015-2019) and PROGEP's Strategic Planning, especially in the use of similar methods and tools in the strategic formulation process, such as Strategic Maps and Balanced Scorecards. Strategic maps have shown links between global and sectoral goals for the development of the institutional philosophy for the field of people management.
\end{abstract}

Keywords: Public Administration. Strategic Consistency. Institutional Alignment. 


\section{INTRODUÇÃO}

O cenário gerencial das organizações passa por contínuas modificações, principalmente nas últimas décadas com o avanço meteórico da tecnologia e a sofisticação de ferramentas administrativas, que contribuem para tornar esse ambiente contingencial e altamente competitivo. No intento de assegurar eficiência e eficácia na oferta de serviços prestados à sociedade, as instituições públicas buscam adotar instrumentos estratégicos visando alcançar objetivos e metas.

As complexidades que permeiam as universidades públicas exigem e criação de instrumentos estratégicos e o encadeamento entre eles, para promover o processo de desdobramento das estratégias para os demais níveis, agregando valor à organização. De igual relevância é a sinergia entre as unidades que apoiam a estratégia organizacional, promover correta alocação de recursos, definição de prioridades, tendo como foco o alcance dos objetivos organizacionais (KAPLAN; NORTON, 2006).

As metodologias e técnicas desenvolvidas no processo de formulação do planejamento estratégico acenam com soluções promissoras, para equacionar a oferta de políticas e práticas de gestão na esfera pública e contribuir para prestação de serviços mais eficientes, eficazes e efetivos à comunidade acadêmica e à sociedade em geral. Trata-se de implantar um processo inovador e subordinado à estratégia organizacional, visando integrar pessoas e suas competências às necessidades da instituição, conciliando os objetivos de ambas as partes (LEITE; ALBUQUERQUE, 2009).

Diante da responsabilidade de produzir conhecimento e formar cidadãos qualificados e atuantes, as universidades federais requerem dos gestores mais proatividade, visão sistêmica e holística e aptidões para planejamento, direção e controle dos processos (MIZAEL et al., 2013). A partir de 2004, as IFES passaram a adotar o Plano de Desenvolvimento Institucional (PDI) como instrumento de planejamento estratégico, por possuir filosofia global e concatenar objetivos, metas, prazos e alcance de resultados.

O PDI da Universidade Federal do Amapá (UNIFAP), que está em vigência, foi construído no início de 2015, para o quinquênio de 2015-2019. Nesse instrumento estratégico, foram alocadas políticas relativas ao ensino, pesquisa e extensão, tríade norteadora das instituições de ensino superior brasileiras, elencadas as diretrizes e ações de gestão para áreas administrativas, financeiras e as relacionadas com desenvolvimento, capacitação e avaliação de pessoal (UNIFAP, 2015). 
Essa dinâmica organizacional das universidades, sobretudo as públicas brasileiras, somada a iminente necessidade de delimitar objetivos estratégicos, direcionar ações, mensurar resultados e discutir formalmente políticas de gestão de pessoas, oportunizou a elaboração do planejamento estratégico da Pró-Reitoria de Gestão de Pessoas (PROGEP), que ocorreu no início do ano de 2016, com vigência de quatro anos (2016-2019), ancorado em objetivos estratégicos do PDI.

Esse contexto instigou a questão de partida desta pesquisa, a saber: Como se estabelecem as relações de alinhamento estratégico entre o Plano de Desenvolvimento Institucional (PDI) e o planejamento estratégico de gestão de pessoas da Universidade Federal do Amapá? Para desvelar essa incógnita, o objetivo geral deste estudo incidiu em identificar o alinhamento entre o plano de desenvolvimento institucional e o planejamento estratégico de gestão de pessoas da Universidade Federal do Amapá.

\section{FUNDAMENTAÇÃO TEÓRICA}

\subsection{GESTÃO ESTRATÉGICA}

A gestão estratégica é uma política originária do processo evolutivo das teorias organizacionais, obtendo maior relevância, tanto em âmbito científico quanto organizacional, a partir da década de 1950, cuja finalidade consiste em sincronizar estratégias, instituições e o meio no qual estão inseridas, por esforço coletivo e sinérgico (MAINARDES; FERREIRA; RAPOSO, 2011). Diante da variabilidade conceitual da palavra estratégia, Mintzberg et al. (2006) apresentam os "Cinco Ps para Estratégia”.

Quadro 1 Cinco Ps para Estratégia

\begin{tabular}{|l|l|}
\hline \multicolumn{1}{|c|}{ Definição } & \multicolumn{1}{c|}{ Descrição } \\
\hline \multirow{2}{*}{ Como Plano } & $\begin{array}{l}\text { Um tipo de curso de ação conscientemente pretendido, uma diretriz (ou } \\
\text { um conjunto de diretrizes) para lidar com uma situação. }\end{array}$ \\
\cline { 2 - 2 } Como Pretexto & "Manobra" específica para superar um oponente ou concorrente. \\
\cline { 2 - 2 } Como Posição & $\begin{array}{l}\text { Um padrão em uma corrente de ação. [...] consistência no comportamento, } \\
\text { pretendida ou não. }\end{array}$ \\
\cline { 2 - 2 } Como Perspectiva & $\begin{array}{l}\text { Um meio de localizar uma organização naquilo que os teóricos } \\
\text { organizacionais gostam de chamar de "ambiente". }\end{array}$ \\
\cline { 2 - 2 } & $\begin{array}{l}\text { Consiste não apenas de uma posição escolhida, mas também de uma } \\
\text { maneira fixa de olhar o mundo. }\end{array}$ \\
\hline
\end{tabular}

Fonte: Adaptado de Mintzberg et al. (2006).

O termo gestão estratégica possui amplitude mais elevada que planejamento estratégico e envolve a elaboração de indicadores de desempenho, a definição de metas e 
ações, que conduzirão ao desenvolvimento organizacional, tendo o planejamento estratégico como principal instrumento (COELHO JUNIOR, 2003; COSTA, 2007).

A estratégia é a expressão da teoria própria da organização e conduz à obtenção de vantagens competitivas, contudo, deve-se compreender que, como outras teorias, é uma sintetização que notadamente desvirtua o objeto da realidade, pois nem sempre representam o real, levando uma informação a ter diversos vieses e efeitos (MINTZBERG; AHLSTRAND; LAMPEL, 2010; BARNEY; HERSTERLY, 2011), especialmente porque há erros de "leitura" dos ambientes interno e externo.

Desse modo, processo de administração estratégica deve reunir métodos de análises, que aumentem a probabilidade de selecionar a estratégia mais vantajosa, obedecendo a certo rito sequencial (BARNEY; HERSTERLY, 2011). A gestão estratégica possui aproximações pragmáticas com o planejamento estratégico. Essa justaposição oportuniza ações estratégicas como o processo de elaboração de planos de ação, gerenciamento do relacionamento entre a liderança e os colaboradores, análise ambiental, controle, avaliação e formação de ambiente propício à inovação (MACÊDO et al., 2012).

\subsection{PLANEJAMENTO ESTRATÉGICO}

$\mathrm{O}$ ato de planejar se tornou ferramenta imprescindível para promover visão holística aos gestores, subsidiando o processo de delimitação dos reais propósitos da organização e a priorização de programas e projetos estratégicos, que contribuem para tornar consonante as ações e o alcance de metas, a partir da missão e dos objetivos organizacionais (MARCELINO, 2002; CAVALCANTE; ROCHA JUNIOR, 2014; SILVA; ASSIS, 2016). Entretanto, o Plano Estratégico resultante do processo de planejar não pode ser rígido, já que os ambientes interno e externo sofrem variações enquanto os projetos estão sendo executados.

Para Thompson e Strickland III (2000), o processo de formulação do planejamento possui cinco etapas fundamentais: $\left(1^{\mathrm{a}}\right)$ definição estratégica da missão, visão e valores da organização; ( $\left.2^{\mathrm{a}}\right)$ estabelecimento dos objetivos; $\left(3^{\mathrm{a}}\right)$ formulação das estratégias; $\left(4^{\mathrm{a}}\right)$ implementação das estratégias; $\left(5^{\mathrm{a}}\right)$ avaliação e controle do desempenho. Esses elementos devem reunir aspectos e diretrizes relevantes da organização, pois eles orientarão à elaboração das prioridades, formando a real imagem que a organização busca propagar no mercado e no meio social (SOUZA; MARINHO, 2014; RIBEIRO; CARMO, 2015). 
O planejamento estratégico moderno se utiliza do auxílio de diversas ferramentas: análise SWOT (Strenghs, Weakness, Opportunities, and Threats), BSC (Balanced scorecard), $5 w 2 h$ e a ferramenta de gerenciamento de projetos PMBOK - Project Management Book of Knowledge (CUNHA et al., 2011; OLIVEIRA; TODA, 2013).

Os estudos sobre Balanced Scorecard (BSC) datam de 1990, objetivando a criação de um modelo inovador de mensuração de desempenho, que agregasse valor econômico projetado para o futuro, pois as mudanças do contexto mercadológico tornavam obsoletos os indicadores de caráter tão-somente contábeis e financeiros, cujo foco concentrava-se apenas no curto prazo (KAPLAN; NORTON, 1997).

Essa ferramenta atua justamente no equilíbrio estrutural de objetivos de curto e longo prazo, organizando-os sob quatro perspectivas: financeira, dos clientes, de processos internos, de aprendizado e crescimento (KAPLAN; NORTON, 1997). Assim, "cada uma das quatro perspectivas é interligada por uma cadeia de relações de causa e efeito" (KAPLAN; NORTON 2006, p. 07).

Mintzberg, Ahlstrand e Lampel (2010) reúnem dez escolas que embasam teoricamente o processo de formulação de estratégia, o estudo aponta contribuições, limitações e críticas de cada uma delas, cuja atuação enfatiza perspectivas diferenciadas e relevantes para o complexo organizacional.

As três escolas iniciais - Design, Planejamento e Posicionamento - possuem natureza prescritiva, focalizando mais os procedimentos de formulação das estratégias, do que o entendimento de como elas realmente se formam, enquanto que as seis escolas imediatas Empreendedora, Cognitiva, Aprendizado, Poder, Cultural, Ambiental - possuem natureza descritiva e buscam compreender aspectos peculiares do processo de formulação, relacionados com o entendimento de como as estratégias realmente se desenvolvem; e finalmente, a escola de Configuração, que reúne as características de todas as anteriores e agrupa variáveis relevantes da existência da organização, a saber: o processo de formação estratégica, seu conteúdo, o arcabouço organizacional e suas conjunturas (MINTZBERG; AHLSTRAND, LAMPEL, 2010).

Desse modo, o cenário de mudança estrutural no gerenciamento da administração pública exige percepções diferenciadas sobre a necessidade de elaboração do planejamento estratégico (PAULI; BIULCHI, 2012). Nessa esfera, a necessidade de priorizar ações que 
visem à melhoria dos serviços prestados à sociedade, tem ganhado destaque na literatura envolvendo a discussão do planejamento estratégico (SILVA; GONÇALVES, 2011).

Ao ser elaborado no setor público, o planejamento estratégico exige que sejam consideradas todas as peculiaridades coexistentes. Contudo, os aspectos inovadores desse processo ainda são tratados como emergentes, tanto nas ações empregadas nos órgãos públicos, quanto no debate teórico de conceituação (SANTOS; SANO, 2016).

Dentre as principais peculiaridades que permeiam a esfera pública, destacam-se o ambiente cheio de complexidades, as influências de viés político, a gestão com autonomia reduzida e a baixa oferta de políticas de pessoal (WRIGHT; KROLL; PARNELL, 2000; SILVA, 2016). Esse contexto torna o planejamento estratégico imprescindível para melhorar o andamento da atividade estatal, devendo instituições públicas seguir as estratégias traçadas, acompanhando a execução, para medir e comparar os resultados alcançados (ALVES et al., 2013; BRANCO, 2014).

\subsection{PLANO DE DESENVOLVIMENTO INSTITUCIONAL}

As universidades federais brasileiras gerenciam diversos processos organizacionais concentrados especialmente em três frentes estratégicas: ensino, pesquisa e extensão (DAL MAGRO; RAUSCH, 2012). Essas políticas devem ser executadas em conformidade com requisitos da legislação específica dessas instituições e cumprir exigências do Ministério da Educação (MEC), o que demanda ações estratégicas de planejamento, remodelamento de processos e avaliação contínua dos processos institucionais (GUEDES; SCHERER, 2015; MIZAEL et al., 2013).

A criação das Instituições Federais de Ensino Superior (IFES) foi marcada pela edição do Decreto-Lei $n^{\circ}$ 200, de 1964, que visou atribuir maior eficiência à atividade estatal, descentralizando funções concorrentes com a iniciativa privada, exigindo posturas estratégicas, práticas inovadoras com foco no cliente-cidadão e inserção da cultura do planejamento (MIZAEL et al., 2013; BRESSER-PEREIRA, 1998).

Nesse contexto, o plano de desenvolvimento institucional (PDI) passou a ser utilizado pelas IFES como instrumento estratégico a partir do ano de 2004, com a edição da Lei $n^{\circ}$ 10.861/2004, cujo processo de formulação consiste na exigência de elementos claros, coesos e objetivos, permitindo a interligação entre todos os eixos previamente traçados, tornando-se um instrumento viável estrategicamente para as instituições (MEC, 2004). 


\section{ALINHAMENTO ESTRATÉGICO DE PLANEJAMENTOS INSTITUCIONAIS: ESTUDO EM UMA \\ UNIVERSIDADE FEDERAL DA AMAZÔNIA SETENTRIONAL \\ DOI: http://dx.doi.org/10.5007/1983-4535.2020v13n2p183}

Com a edição do Decreto $\mathrm{n}^{\mathrm{o}} 5.773 / 2006$, o PDI se tornou requisito obrigatório para o ato de credenciamento das IFES junto ao MEC. Os procedimentos obrigatórios foram ampliados, cujos principais eixos temáticos são: (I) Perfil Institucional; (II) Projeto Pedagógico Institucional - PPI; (III) Cronograma de Implantação e Desenvolvimento da Instituição e dos Cursos (presencial e a distância); (IV) Perfil do Corpo Docente; (V) Organização Administrativa da IES; (VI); Políticas de Atendimento aos Discentes; (VII) Infraestrutura; (VIII). Avaliação e Acompanhamento do Desenvolvimento Institucional; (IX) Aspectos Financeiros e Orçamentários; (X) Anexos.

O Decreto $n^{\circ} 5.773 / 2006$ foi revogado pelo Decreto $n^{0} 9.235 / 2017$, que por sua vez expandiu os requisitos indispensáveis do Plano de Desenvolvimento Institucional das universidades. Para o MEC, O PDI está intrinsecamente ligado às ações estratégicas e aos resultados da avaliação da instituição, na forma de processo avaliativo interno e externo.

\subsection{ALINHAMENTO ESTRATÉGICO}

O nível de alinhamento de sistemas como estratégias organizacionais, objetivos globais e outros fatores que compõem o complexo organizacional, contribui diretamente para alcançar status de eficácia no processo de implementação das estratégias organizacionais (BARROS, 2007).

Desse modo, quanto mais alinhadas e claramente definidas as estratégias, os recursos corretamente gerenciados e todos seguindo na mesma direção, mais próxima estará a organização de implantar com sucesso suas estratégias (FORONI, 2014). No nível corporativo, as estratégias são esforços organizacionais para obtenção de vantagens competitivas em diferentes mercados ou nichos, sendo que as estratégias mais comuns atuam de forma integrada e vertical (BARNEY; HERSTERLY, 2011).

O conceito de alinhamento parte do pressuposto de que a estratégia é o núcleo do modelo de gestão (KAPLAN; NORTON, 2006). Os referidos autores consideram que o alinhamento estratégico organizacional se materializa quando são observados quatro aspectos:

$\left.1^{\circ}\right)$ Consistência estratégica: é identificada quando o grupo de norteadores do desempenho interno encontra-se alinhado aos resultados e ao desempenho financeiro almejados, por meio dos mecanismos do Mapa Estratégico.

$\left.2^{\circ}\right)$ Alinhamento organizacional: apoia-se na sinergia entre os diversos componentes organizacionais. Os Mapas Estratégicos e Balanced Scorecards permitem a comunicação entre os diversos níveis organizacionais. 
$\left.3^{\circ}\right)$ Alinhamento do capital humano: é obtido no momento em que os objetivos individuais estiverem alinhados com os organizacionais.

$\left.4^{\circ}\right)$ Alinhamento dos sistemas de planejamento e controle: ocorre quando o sistema de gestão, voltado para planejamento, operações e controle, está sincronizado com a estratégia organizacional.

No modelo teórico proposto por Kaplan e Norton (2006), pode-se identificar a construção de alinhamento a partir do processo de planejamento corporativo, planejamento das unidades de negócio e verificação do sincronismo alocado em pontos estratégicos do fluxo de informações entre as estratégias. Posteriormente à elaboração da estratégia corporativa, as unidades de negócio e de apoio devem elaborar seus planejamentos alinhados com o scorecard organizacional, de forma a manter o equilíbrio estratégico e executar suas metas com maior eficiência (KAPLAN; NORTON, 2006).

\section{PERCURSO METODOLÓGICO}

Visando garantias de segurança e respaldo legal, este estudo foi submetido ao Comitê de Ética em Pesquisa - CEP, da Universidade Federal do Amapá, via Plataforma Brasil, aprovado via Parecer Consubstanciado no 3.180.664, nos moldes da Resolução $n^{\circ}$ 466/2012, do Conselho Nacional de Saúde.

Quanto aos objetivos, este estudo se classifica como exploratório e explicativodescritivo. O viés exploratório visa ampliar e aproximar a percepção sobre um fenômeno, por meio de revisão literária e discussão com outros estudiosos, enquanto que a pesquisa explicativa concentra-se em constatar os potenciais elementos que concorrem para a ocorrência de determinado fato (GIL, 2008). A pesquisa descritiva visa descobrir situações, descrever eventos ou transcrever opiniões que ocorrem em determinada população ou subgrupos (PINSONNEAULT; KRAEMER, 1993).

A abordagem ao problema desta pesquisa é predominantemente qualitativa. Esse enfoque é recomendado para trabalhos que buscam estudar a forma que pessoas e grupos percebem a realidade e formulam conceitos, deixando transparecer, na subjetividade, artefatos sociais desconhecidos (FONTANELLA; RICAS; TURATO, 2008; CAVALCANTE; CALIXTO; PINHEIRO, 2014).

O estudo foi desenvolvido na Universidade Federal do Amapá (UNIFAP), localizada ao norte do Brasil, no Estado do Amapá. Atualmente, a UNIFAP comporta uma estrutura física distribuída em quatro campi, os quais são: Marco Zero, Mazagão, Santana e Binacional 
(UNIFAP, 2019). O lócus deste estudo foi a Pró-Reitoria de Gestão de Pessoas - PROGEP, criada pela Resolução $n^{0}$ 01/2013, do Conselho Diretor da Universidade, e possui atualmente 43 servidores, sendo somente técnicos administrativos de níveis médio e superior (UNIFAP, 2019).

A seleção estratégica dos participantes baseou-se na relevância do seu papel no âmbito institucional e na participação no processo de elaboração dos instrumentos estratégicos, sendo: a reitora da universidade, o pró-reitor de gestão de pessoas, dois diretores departamentais e quatro servidores da categoria dos técnicos administrativos. Os nomes dos entrevistados foram codificados em "GR" para os gestores e TAD para os técnicos administrativos.

A proposta de coleta de dados foi baseada nos estudos de Alves e Silva (1992), cuja estrutura indagatória semiestruturada foi adaptada da pesquisa de Foroni (2014). A entrevista é a técnica mais indicada em análises de percepção de colaboradores sobre processos e políticas organizacionais, pois proporciona ao pesquisador reunir um número elevado de dados e informações que atribuirão relevância ao trabalho científico (BRITTO JUNIOR; FERES JUNIOR, 2011; FARIA, 2015).

Algumas entrevistas com gestores foram realizadas por meio do recurso de vídeochamada da ferramenta Skype. Os depoimentos foram transcritos no aplicativo de web denominado oTranscribe. Os dados coletados foram submetidos à Análise de Conteúdo (AC), difundida nos estudos de Bardin (1977). Dentre os documentos analisados, cita-se: o Plano de Desenvolvimento Institucional da UNIFAP (PDI 2015-2019), o planejamento estratégico da PROGEP e o relatório de gestão.

Para atribuir validade ao método de análise, utilizou-se o auxílio do software ATLAS.ti 8 - Qualitative Data Analysis. O uso deste software é recomendado em pesquisas qualitativas, pois propicia ao pesquisador tratar elevado volume de dados, subsidiando conclusões e inferências acerca do produto extraído (SANTOS, 2018).

\section{ANÁLISE E DISCUSSÃO DOS RESULTADOS}

\subsection{PLANO DE DESENVOLVIMENTO INSTITUCIONAL DA UNIFAP}

Assim como em outras universidades federais, o PDI é considerado o documento formal de planejamento estratégico da Universidade Federal do Amapá, cujos eixos temáticos, estruturalmente, atendem aos requisitos mínimos recomendados pelo Decreto $n^{0}$ 9.235/2017. 


\section{ALINHAMENTO ESTRATÉGICO DE PLANEJAMENTOS INSTITUCIONAIS: ESTUDO EM UMA \\ UNIVERSIDADE FEDERAL DA AMAZÔNIA SETENTRIONAL \\ DOI: http://dx.doi.org/10.5007/1983-4535.2020v13n2p183}

A metodologia utilizada para concatenar a missão, visão, valores, metas e objetivos, é o Balanced Scorecard (BSC). As diretrizes estratégicas da Universidade foram diluídas nas perspectivas sociedade, processos internos, aprendizado e crescimento e orçamento, formando o Mapa Estratégico da Universidade, com 21 objetivos estratégicos. Pode-se compreender melhor a materialização das estratégias institucionais da UNIFAP por meio do fluxo do processo de formação estratégica.

Figura 1 Processo de formulação estratégica da UNIFAP

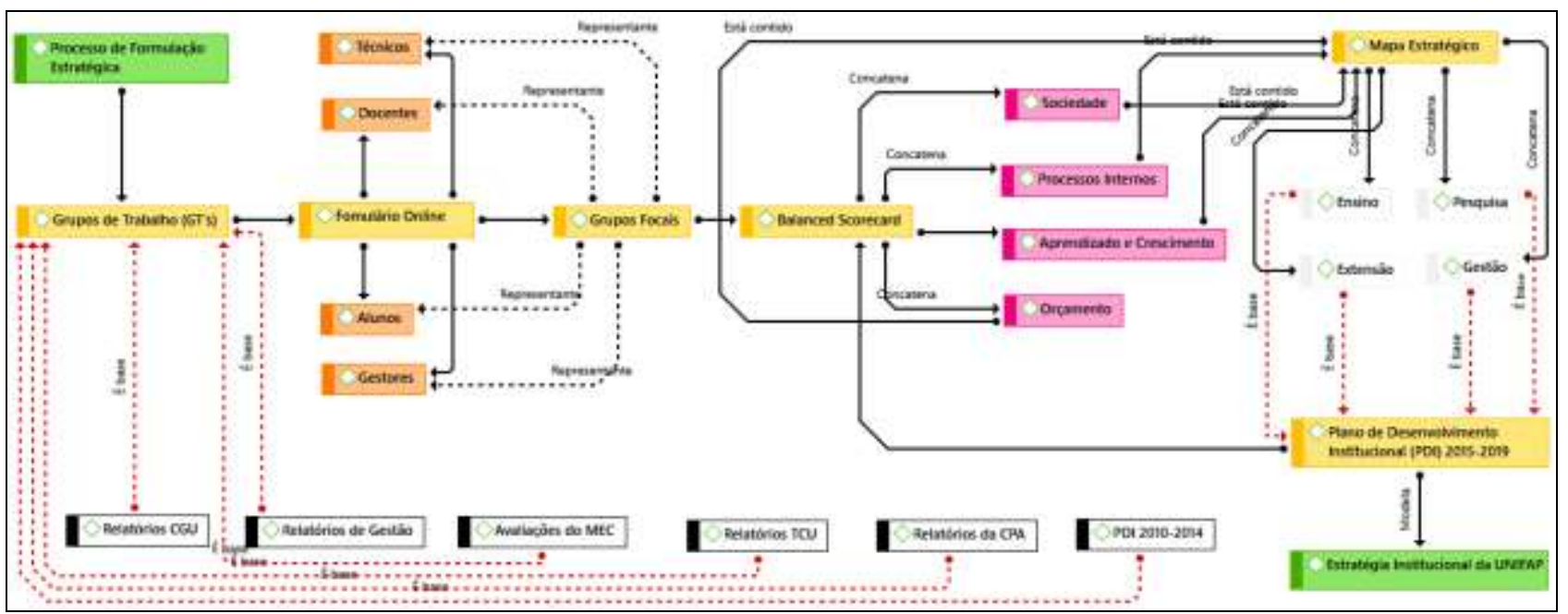

Esse construto mostra que o processo estratégico se iniciou com a formação de grupos de trabalho (GT's), que estruturaram um documento com base em relatórios de órgãos de controle, avaliações do Ministério da Educação (MEC) e relatórios internos da universidade dos últimos oito anos e resgatando ainda informações do PDI 2010-2014. O resultado das análises do perímetro inicial do PDI (2015-2019) e o depoimento do gestor "GR01" convergem quanto à consciência da relevância de envolver os atores que formam a chamada comunidade acadêmica - alunos, técnicos administrativos e docentes - desde as etapas iniciais de discussão.

GR01 - O PDI é um documento que precisa ser organizado tendo a participação da comunidade acadêmica, $[. .$.$] porque ele tem que expressar$ o sentimento dessa comunidade e como ela se projeta ao longo de seu desenvolvimento. Esse sentimento é importante, porque é a partir dele que a gente pode elaborar as estratégias, para alcançar os objetivos e as metas.

Ao se verificar a forma e o momento da participação da comunidade, tendo por base a citação supramencionada e o fragmento inicial do referido documento, deduz-se que as estratégias institucionais seriam delimitadas a partir dos resultados advindos dos debates e 
discussões com a comunidade acadêmica, ainda, que a participação desses agentes seria direta e desde as etapas iniciais.

No entanto, os depoimentos de alguns servidores e gestores vão na contramão ao demonstrar desconhecimento da forma como foi elaborado o PDI (2015-2019) da UNIFAP, algo contraproducente para um processo de gestão estratégica, que deveria ser construído de forma participativa e democrática juntamente com os atores envolvidos.

GR02 - Não participei em nada do PDI da universidade. Na realidade, ele só serviu para dar o norte para o planejamento estratégico da PROGEP.

GR 03 - [...] a gente começou a fazer os planejamentos estratégicos de cada Pró-Reitoria, foi então que a gente começou a ter conhecimento do PDI, inclusive isso foi uma opinião muito forte minha na época, que tivesse sido "coletivo", as Pró-Reitorias não sabiam como funcionava o PDI.

TAD02 - Quanto ao PDI, [...] penso que alguma unidade vinculada à reitoria deve ter enviado expedientes, mas eu não sei como foi elaborado o processo. Nem sei se os objetivos foram alcançados, não há esse feedback.

TAD04 - Na verdade eu não participei desse processo, ele foi aberto, mas não foi amplamente divulgado para participação da comunidade de forma geral. [...] foi um instrumento gerado de forma centralizada.

Assim, torna-se evidente certa deficiência no processo de participação da comunidade acadêmica na construção das estratégias institucionais. A participação social, em cada fase do processo de planejamento estratégico em universidades públicas, é fundamental para que a comunidade compreenda na íntegra as ações, os planos de trabalho e todas as informações relevantes para a implementação das estratégias e permite a continuidade administrativa (SANTOS et al., 2009).

Sob a ótica dos "Cinco Ps para Estratégia" de Mintzberg et al., (2006), as estratégias do PDI da UNIFAP podem ser vistas como: "Plano", pois se resumem na delimitação de uma gama de diretrizes e ações norteadoras para alcançar objetivos institucionais; "Padrão", pois a estratégia deve seguir um rito previamente definido pela legislação e atender exigências de órgãos governamentais, MEC, o que limita a atuação da gestão institucional no processo de formulação estratégica; ainda, como "Posição", pois situa a instituição no espaço e define o seu papel diante das peculiaridades ambientais.

No contexto teórico das dez escolas de planejamento, o Plano de Desenvolvimento Institucional da UNIFAP possui características similares com a "Escola de Planejamento", 
cuja formulação estratégica é concebida como um processo "Formal" (MINTZBERG; AHLSTRAND, LAMPEL, 2010).

\subsection{PLANEJAMENTO ESTRATÉGICO DE GESTÃO DE PESSOAS}

A necessidade de elaboração do planejamento de gestão de pessoas tornou-se ainda mais latente após a formulação do Plano de Desenvolvimento Institucional - PDI (20152019), diante da necessidade de desdobramento das estratégias institucionais para o campo da gestão de pessoas, de maneira alinhada. Cabe ressaltar, que as políticas e práticas de gestão de pessoas no setor público são delimitadas geralmente nas esferas governamentais, expressadas por meio de legislação, neste caso, pela Lei $\mathrm{n}^{0}$ 11.091/2005 (PCCTAE) e pelo Decreto $\mathrm{n}^{\circ}$ 5.707/2006 (PNDP). O fluxo do processo de formação estratégica demonstra e corrobora as nuances das variáveis que serviram de base para a gestão estratégica de pessoas da UNIFAP.

Figura 2 Processo de formulação da gestão estratégica de pessoas

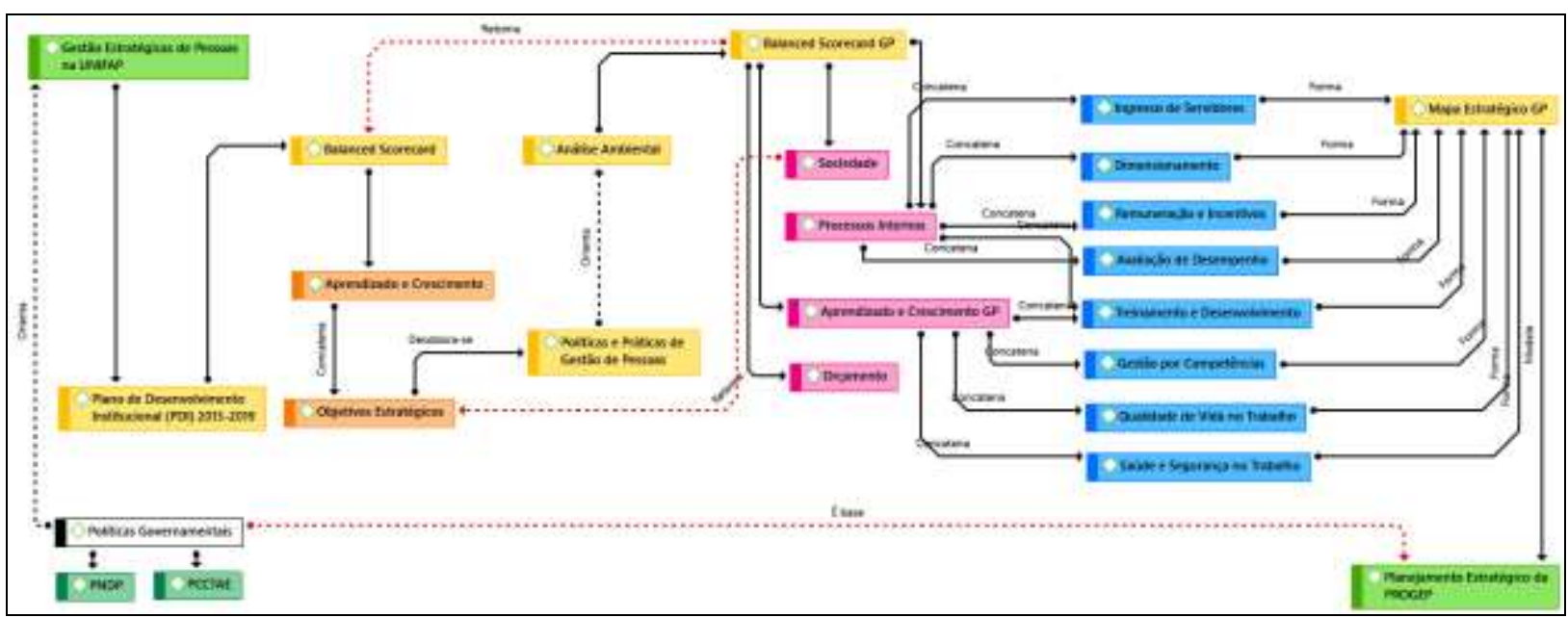

O fluxo da figura ilustra que a gestão estratégica de pessoas da UNIFAP foi orientada inicialmente por políticas governamentais, especialmente representadas pelo PNDP e PCCTAE, ancorada ao PDI (2015-2019) por meio de objetivos estratégicos. O processo de planejamento da PROGEP foi construído em parceria com professores e bolsistas do curso de administração da UNIFAP, como projeto de extensão, promovendo capacitação aos servidores da Pró-Reitoria, onde também foi apresentado o PDI (2015-2019) e as metodologias a serem utilizadas.

Segundo os entrevistados, houve participação direta da equipe de servidores da PROGEP nas reuniões para o processo de elaboração do planejamento estratégico. Como 
qualquer outra forma de implementação estratégica, o alinhamento exige que as equipes envolvidas no processo estejam integradas e atuem de forma cooperativa para alcançar os objetivos organizacionais (KAPLAN; NORTON, 2006). Esse achado converge com os resultados dos estudos de Silva et al., 2017.

Os objetivos estratégicos foram concatenados nas quatro perspectivas do BSC (sociedade, processos internos, aprendizado e crescimento, orçamento), formando o mapa estratégico da PROGEP. Os objetivos foram desdobrados para as atividades dos planos de ação de cada divisão administrativa, com fixação de indicadores e metas. O uso de metodologias similares demonstra um ponto essencial para promoção de alinhamento entre o planejamento estratégico RH e o PDI (2015-2019) da UNIFAP.

A partir do resgate teórico deste estudo, nota-se que o planejamento estratégico da PROGEP pode ser enquadrado dentro das perspectivas da "Escola de Planejamento", por consistir em um processo "Formal" de formulação estratégica, o qual utilizou ferramentas como o $B S C, S W 2 H$ e Mapas Estratégicos, assim como possui similaridades com a "Escola Cultural", cuja formulação estratégica é concebida como um "Processo Coletivo", uma vez que houve integração social nas etapas de elaboração do planejamento, que considerou percepções, valores e crenças da equipe que compõe a Pró-Reitoria (MINTZBERG; AHLSTRAND, LAMPEL, 2010).

\subsection{ALINHAMENTO DE MAPAS ESTRATÉGICOS INSTITUCIONAIS}

A análise dos mapas estratégicos das duas ferramentas foi fundamental para a construção do fluxo a seguir, cujo conteúdo demonstra como se materializaram os principais encadeamentos e nuances entre o Plano de Desenvolvimento Institucional - PDI (2015-2019) e o Planejamento Estratégico da PROGEP, por meio de um processo de comparabilidade dos ritos instrumentais e metodológicos, interligados por elementos basilares e associativos.

Verifica-se que existe simetria entre os mapas estratégicos dos instrumentos de planejamento, inclusive com arranjos estruturais semelhantes, iniciando na definição das diretrizes estratégicas - missão, visão e valores - com associações e encadeamentos, bem como convergência na ordem dimensional.

Essa organização do complexo organizacional, traduzida em objetivos estratégicos globais e setoriais, metas e indicadores, favorece a eficácia no processo de implementação e 
controle das estratégias definidas, uma vez que tendem a caminhar na mesma direção (BARROS, 2007; FORONI, 2014; SANTOS; NASCIMENTO; OLIVEIRA, 2018).

Figura 3 Encadeamento entre mapas estratégicos

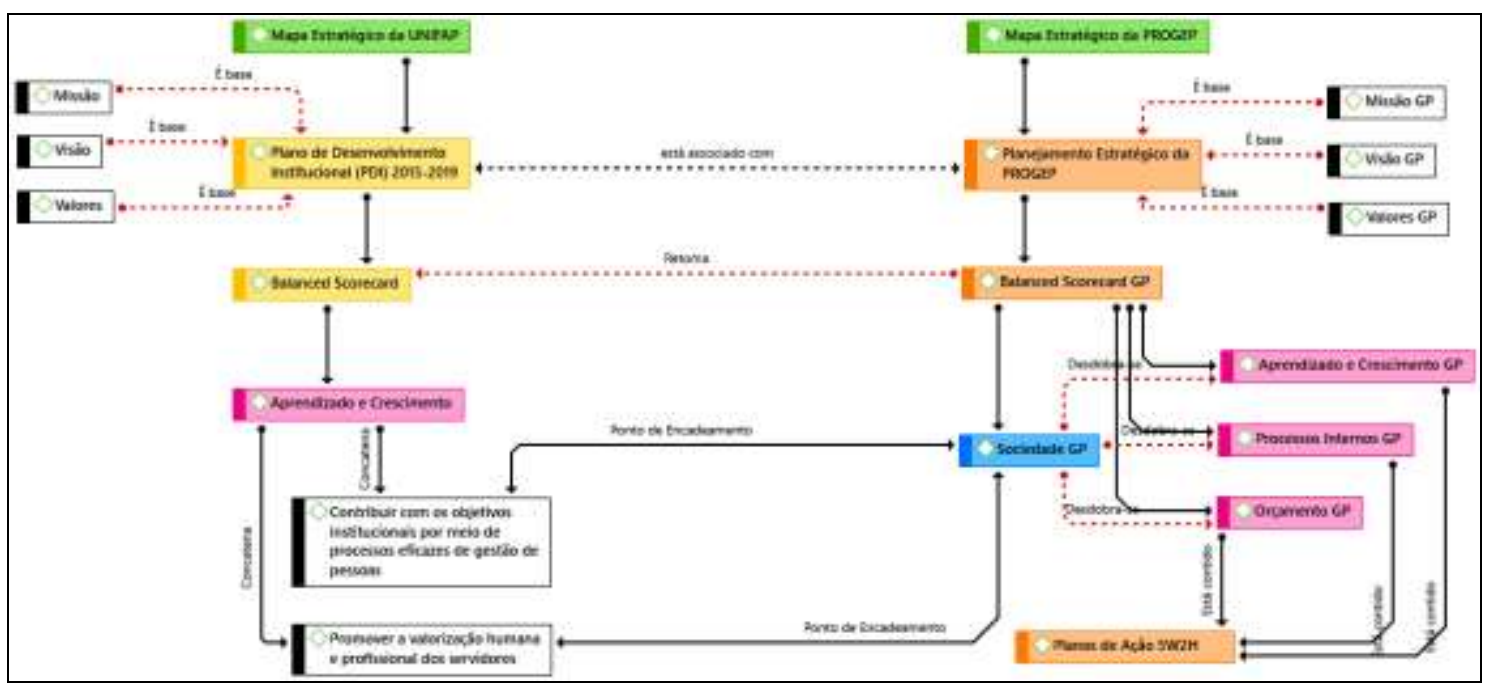

O ponto de encadeamento ocorreu, a partir de dois objetivos estratégicos globais situados na perspectiva Aprendizado e Crescimento do BSC do PDI (2015-2019), os quais foram alocados na dimensão Sociedade do $B S C$ do Planejamento Estratégico de Gestão de Pessoas, conforme demonstrado no fluxo acima, permitindo o desdobramento da filosofia global para as unidades setoriais, onde foram concatenadas as políticas e práticas de gestão de pessoas, distribuídas posteriormente nos planos de ação $(5 \mathrm{~W} 2 \mathrm{H})$.

Retomando o cotejamento teórico de Kaplan e Norton (2006), verificou-se que: (a) tanto o PDI (2015-2019) quanto o Planejamento Estratégico da PROGEP possuem "consistência estratégica", pois a estratégia delineada possui objetivos estratégicos e indicadores bem definidos, fator positivo na etapa de implementação e avaliação; (b) pode-se considerar que ambos os instrumentos atendem ao aspecto "Alinhamento Organizacional", pois se apoiam nos mecanismos fornecidos pelo Mapa estratégico e Balanced Scorecard, especialmente para comunicar as estratégias para os demais níveis organizacionais; (c) quanto ao "alinhamento do capital humano", somente o Planejamento da PROGEP atende a esse aspecto em sua plenitude, pois foi elaborado de forma participativa, o que propicia a compreensão das estratégias traçadas, enquanto que o PDI (2015-2019) atende parcialmente, pois a maioria dos sujeitos entrevistados demonstrou não conhecer efetivamente 0 instrumento e não participaram de sua elaboração; (d) no que se refere ao "alinhamento dos 
sistemas de planejamento e controle", verificou-se que ambos os instrumentos atendem esse aspecto, pois são utilizados formalmente para delinear as estratégias e apoiam-se no auxilio dos relatórios de gestão, que acompanham os indicadores para mensurar o desempenho institucional.

\subsection{ALINHAMENTO À LUZ DO MODELO DE KAPLAN E NORTON}

O arranjo de análise demonstrado na figura a seguir, expõe o alinhamento do processo de planejamento estratégico da UNIFAP, a partir das nuances subsistentes no ambiente interno da instituição, de modo multidirecional - alinhamento vertical e horizontal. O alinhamento vertical está relacionado com a configuração das estratégias, objetivos, planos de ação e decisões nos diversos níveis organizacionais, enquanto que o alinhamento horizontal pode ser definido em termos de integração entre as funções e dentro das funções (HEINZEN; MARINHO, 2019).

Figura 4 Alinhamento no processo de planejamento UNIFAP/PROGEP

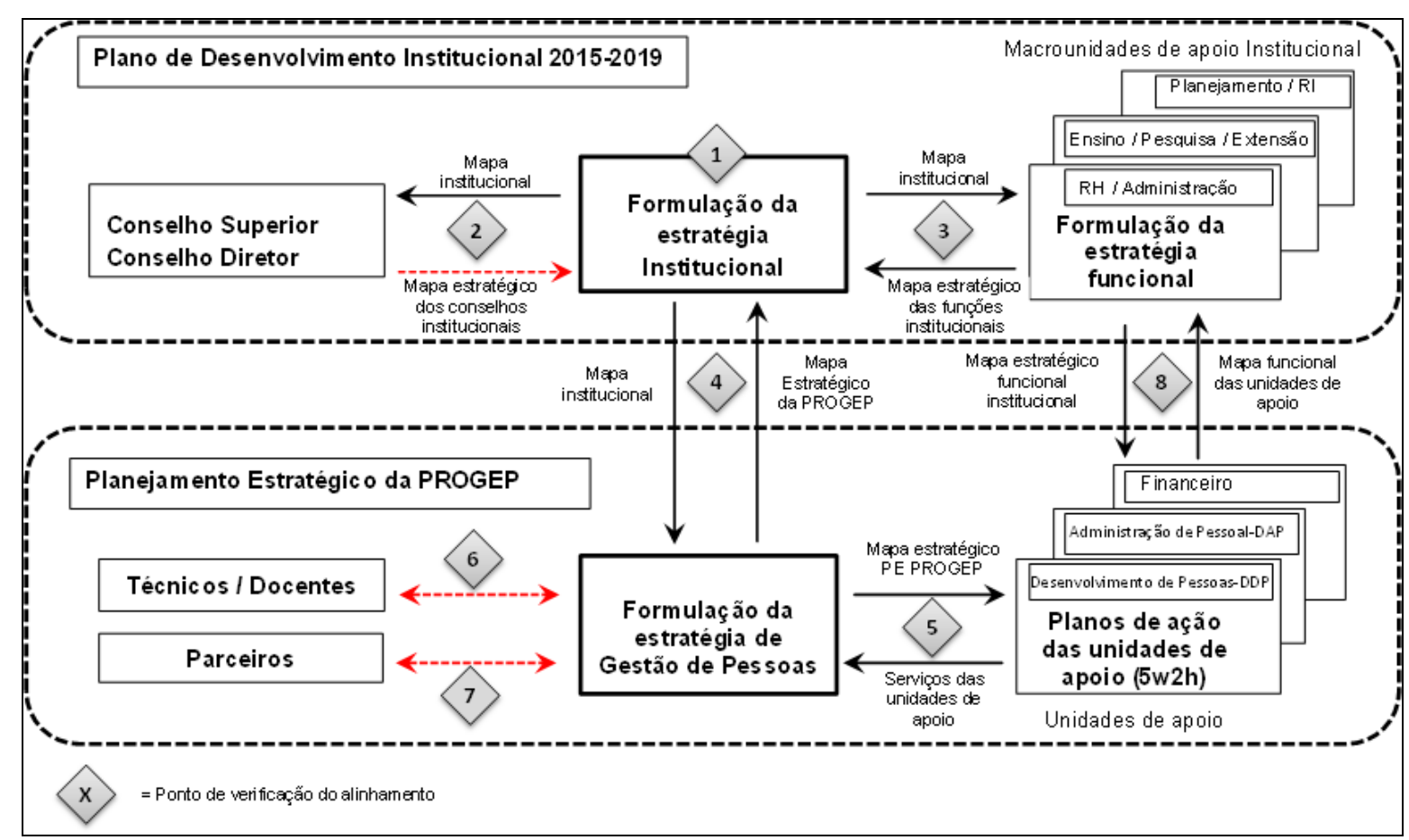

Fonte: Adaptado do modelo de Kaplan e Norton (2006), elaborado pelo autor.

Dentre os principais marcos, o ponto de verificação 1, epicentro da estratégia institucional, localizam-se as diretrizes institucionais e as políticas alocadas no mapa estratégico. A proposta de valor da UNIFAP encontra-se no mapa estratégico do Plano de 
Desenvolvimento Institucional - PDI (2015-2019), onde estão concatenadas missão, visão, valores e objetivos estratégicos, que representam as políticas institucionais.

O ponto de verificação 3, por exemplo representa a tradução da estratégia institucional em políticas e normatizações que serão gerenciadas pelas demais unidades da instituição (KAPLAN; NORTON, 2006). Esse alinhamento é um dos processos mais relevantes no cenário estratégico da UNIFAP, pois as políticas envoltas no tripé ensino, pesquisa e extensão, políticas de recursos humanos, administrativas, financeiras e de relações internacionais são concatenadas no mapa estratégico do PDI (2015-2019), em forma de objetivos estratégicos, para permitir o desdobramento das estratégias institucionais para as diversas macrounidades da Universidade, que são as Pró-Reitorias.

$\mathrm{Na}$ UNIFAP, o ponto de verificação 4 representa o alinhamento entre a estratégia institucional e seu desdobramento para o campo da gestão de pessoas da UNIFAP, cuja proposta de valor são as políticas e práticas de gestão de pessoas. Para Kaplan e Norton (2006), este ponto de verificação representa o desdobramento das prioridades institucionais em estratégias funcionais, que serão desenvolvidas pelas macrounidades da organização.

No ponto de verificação 5 , as prioridades desdobradas para as macrounidades são agrupadas nas estratégias executadas pelas unidades de apoio funcionais (KAPLAN; NORTON, 2006). Na UNIFAP, é possível verificar que o processo de formulação das estratégias da PROGEP distribui as políticas e práticas de gestão de pessoas para as unidades de apoio, representadas pelos departamentos e divisões, que elaborarão planos de ação para executar essas políticas e práticas.

O ponto de verificação 8 demonstra que as estratégias executadas pelas unidades de apoio também possuem relação reflexa com as prioridades das outras áreas de atuação organizacional (KAPLAN; NORTON, 2006). Assim, é perceptível que políticas e práticas de gestão de pessoas da PROGEP têm papel estratégico e relevante no auxílio das atividades desenvolvidas pelas outras Pró-Reitorias da UNIFAP, ajudando a disseminar a filosofia de gestão de pessoas no âmbito institucional.

Nesse aspecto, "usando esses oito pontos de verificação como referência, as organizações podem medir e gerenciar o grau de alinhamento e, consequentemente, aumentar a sinergia na organização" (KAPLAN; NORTON, 2006, p. 18). 


\section{CONSIDERAÇÕES FINAIS}

O enredo deste estudo consistiu em responder ao seguinte objetivo geral: identificar o alinhamento entre o plano de desenvolvimento institucional e o planejamento estratégico de gestão de pessoas da Universidade Federal do Amapá.

Constatou-se, que as ferramentas utilizadas para modelar o escopo estratégico dos instrumentos de planejamento institucional foram o Balanced Scorecad e Mapas Estratégicos, análise SWOT e planos de ação (5w2h). No nível estratégico, foi constatado que o Plano de Desenvolvimento Institucional - PDI é utilizado como documento formal de planejamento.

Como pontos que necessitam de ações corretivas, destacam-se: a ausência de eixo temático específico para tratar especificamente da gestão estratégica de pessoas, diretamente no PDI (2015-2019) da UNIFAP; desconhecimento por parte dos servidores dos objetivos, metas e ações elencados no referido documento; ausência de ações formais para viabilizar a participação da comunidade acadêmica nas etapas de construção do instrumento estratégico institucional; falta de uma política de comunicação voltada para divulgar ações e resultados alcançados do PDI.

Nos níveis tático e operacional, identificou-se que o Planejamento Estratégico da PROGEP foi elaborado de maneira participativa, cujo produto final representou o sentimento do capital humano da referida Pró-Reitoria, prática que pode ser estendida à elaboração do PDI, visando captar os anseios da comunidade acadêmica. O ponto de encadeamento no alinhamento dos mapas estratégicos aconteceu em dois objetivos estratégicos globais, da perspectiva Aprendizado e Crescimento do BSC do PDI (2015-2019), os quais se interligam com a dimensão Sociedade do BSC do Planejamento Estratégico da PROGEP, desdobrando a filosofia institucional para o campo da gestão de pessoas.

Sobre os pontos de alinhamento no processo estratégico da UNIFAP, entre a formulação do PDI (2015-2019) e do Planejamento Estratégico de RH, do ponto de vista horizontal, constatou-se que a estratégia da UNIFAP é concebida a partir do tripé ensino, pesquisa e extensão, além das atividades de gestão, cujo gerenciamento é realizado pelas macrounidades chamadas Pró-Reitorias. Enquanto que o Planejamento da PROGEP se materializa pelas políticas e práticas de gestão de pessoas, gerenciadas por departamentos e divisões administrativas. $\mathrm{O}$ alinhamento vertical se consolida de forma cíclica, inicialmente entre os mapas estratégicos do PDI (2015-2019) e do planejamento estratégico da PROGEP, e em seguida entre as ações ofertadas pelas divisões da PROGEP para as demais Pró-Reitorias. 
Afirma-se, que o objetivo proposto foi alcançado de forma satisfatória. Sugere-se, que este estudo e outros semelhantes sejam aplicados em outras instituições públicas, visando identificar similaridades ou divergências com os apontamentos aqui levantados. Sugere-se, ainda, outras investigações a partir deste trabalho, que visem identificar os principais desafios enfrentados pelas instituições públicas, para implementação simultânea das estratégias do processo institucional e do planejamento de gestão de pessoas.

\section{REFERÊNCIAS}

ALVES, J. N. et al. A utilização do planejamento estratégico como ferramenta de gestão na pequena empresa. Revista da Micro e Pequena Empresa, v. 7, n. 2, p. 80-100, 2013.

ALVES, Z. M. M. B.; SILVA, M. H. G. F. D. da. Análise qualitativa de dados de entrevista: uma proposta. Paidéia (Ribeirão Preto), [s.1.], n. 2, p.61-69, jul. 1992. FapUNIFESP (SciELO). http://dx.doi.org/10.1590/s0103-863x1992000200007

BARDIN, L. Análise de conteúdo. Ed. 70. Lisboa: Edições, 1977.

BARNEY, J. B.; HESTERLY, W. Administração estratégica e vantagem competitiva: conceitos e casos. 3. ed. São Paulo: Pearson Prentice Hall, 2011. 408 p. Tradução de: Midori Yamamoto.

BARROS, L. A. M. de. Alinhamento estratégico. 2007. Tese (Doutorado em Administração) - Faculdade de Economia, Administração e Contabilidade, Universidade de São Paulo, São Paulo, 2007.

BRANCO, L. S. C. O planejamento estratégico no setor público brasileiro. Revista de Gestão e Contabilidade da UFPI, Floriano, v. 1, n. 1, p.173-182, jun. 2014.

BRASIL. Decreto $\mathrm{n}^{\mathrm{o}}$ 5.707, de 23 de fevereiro de 2006. Institui a política e as diretrizes para o desenvolvimento de pessoal da administração pública federal direta, autárquica e fundacional, e regulamenta dispositivos da Lei ${ }^{\circ}$ 8.112, de 11 de dezembro de 1990. Brasília, DF: Diário Oficial da União, 23 fev. 2006.

BRASIL. Lei no 11.091, de 12 de janeiro de 2005. Dispõe sobre a estruturação do plano de carreira dos cargos técnico-administrativos em educação, no âmbito das instituições federais de ensino vinculadas ao ministério da educação, e dá outras providências. Brasília, DF: Diário Oficial da União, 13 jan. 2005.

BRESSER-PEREIRA, L. C. Uma reforma gerencial da administração pública no Brasil. Revista do Serviço Público, Brasília, v. 49, n. 1, p.5-42, mar. 1998. Trimestral.

BRITTO JUNIOR, A. F. de; FERES JUNIOR, N. A utilização da técnica da entrevista em trabalhos científicos. Revista Evidência, Araxá, v. 7, n. 7, p. 237-250, 2011. 
CAVALCANTE, H. M. B.; ROCHA JUNIOR, C. J. G. Planejamento estratégico para empresas produtoras de sementes de cebola (Allium Cepa L.) no estado de Pernambuco. Interface - Revista do Centro de Ciências Sociais Aplicadas, v. 11, n. 2, p. 29-46, 2014.

CAVALCANTE, R. B.; CALIXTO, P.; PINHEIRO, M. M. K. Análise de conteúdo: considerações gerais, relações com a pergunta de pesquisa, possibilidades e limitações do método. Informação \& Sociedade: Estudos, João Pessoa, v. 24, n. 1, p.13-18, abr. 2014.

COELHO JUNIOR, F. A. Gestão estratégica: um estudo de caso de percepção de mudança de cultura organizacional. Psico: USF, São Francisco, v. 8, n. 1, p.81-89, jun. 2003. Bimestral.

COSTA, E. A. da. Gestão estratégica: da empresa que temos para a empresa que queremos. 2. ed. São Paulo: Saraiva, 2007.

CUNHA, A. S. et. al. Planejamento estratégico: mapeamento das publicações no período de 2000 a 2010. Revista Pensamento Contemporâneo em Administração, v. 5, n. 3, p. 55-65, 2011.

DAL MAGRO, C. B.; RAUSCH, R. B. Plano de Desenvolvimento Institucional de universidades federais brasileiras. Administração: ensino e pesquisa, Rio de Janeiro, v. 13, n. 3, p.427-454, set. 2012. Trimestral.

FARIA, J. H. de. Análise de discurso em estudos organizacionais: as concepções de Pêcheux e Bakhtin. Teoria e Prática em Administração, João Pessoa, v. 5, n. 2, p.51-71, out. 2015.

FONTANELLA, B. J. B.; RICAS, J.; TURATO, E. R. Amostragem por saturação em pesquisas qualitativas em saúde: contribuições teóricas. Cadernos de Saúde Pública, Rio de Janeiro, v. 24, n. 1, p.17-27, jan. 2008.

FORONI, P. G. Gestão estratégica de pessoas: um estudo de caso sobre o alinhamento estratégico. 2014. Dissertação (Mestrado em Administração) - Faculdade de Economia, Administração e Contabilidade, Universidade de São Paulo, São Paulo, 2014.

GIL, A. C. Métodos e técnicas de pesquisa social. 6. ed. São Paulo: Atlas, 2008. 220 p.

GUEDES, E. P.; SCHERER, F. L. O processo de elaboração do Plano de Desenvolvimento Institucional (PDI): um estudo de caso na Universidade Federal do Paraná. Perspectivas em Gestão e Conhecimento, João Pessoa, v. 5, n. 2, p.240-253, dez. 2015. Semestral.

HEINZEN, D. A. M.; MARINHO, S. V. Alinhamento entre Formulação e Implementação da Estratégia em Instituições de Ensino Superior no Brasil. Revista Organizações em Contexto, [s.1.], v. 15, n. 29, p.279-322, 4 fev. 2019.

KAPLAN, R. S.; NORTON, D. P. Alinhamento: utilizando o balanced scorecard para criar sinergias corporativas, Rio de Janeiro: Elsevier, 2006. 
KAPLAN, R. S.; NORTON, D. P. Balanced Scorecard: a estratégia em ação. Rio de Janeiro: Campus, 1997.

LEITE, N. P.; ALBUQUERQUE, L. G. de. Políticas e práticas de gestão de pessoas: peculiaridades de uma estrutura organizacional remota. Revista de Administração da UFSM, Santa Maria, v. 2, n. 2, p.327-342, ago. 2009.

MACÊDO, S. de et al. Planejamento e gestão estratégica: um estudo sobre adoção e práticas em indústrias do Rio Grande do Norte. Sistemas \& Gestão, [s.1.], v. 7, n. 1, p.58-75, 2012. LATEC. http://dx.doi.org/10.7177/sg.2012.v7.n1.a4.

MAINARDES, E. W.; FERREIRA, J.; RAPOSO, M. Conceitos de estratégia e gestão estratégica: qual é o nível de conhecimento adquirido pelos estudantes de gestão?. FACEF Pesquisa, Franca, v.14, n.3, p. 278-298, set./out./nov./dez. 2011.

MARCELINO, G. F. Planejamento estratégico no setor público: uma experiência no palácio do planalto. Revista do Serviço Público, Brasília, v. 53, n. 4, p.107-124, dez. 2002.

MEC. Ministério da Educação. Plano de desenvolvimento institucional - PDI: diretrizes para elaboração. 2004. Elaborada pelo SAPIENS/MEC, com base na Lei n ${ }^{0} 10.861$, de 14 de abril de 2004. Disponível em: https://www.dti.ufv.br/cpa/doc/pdi sapiens.pdf . Acesso em: 29 abr. 2019.

MINTZBERG, H. et al. O processo da estratégia: conceitos, contextos e casos selecionados. 4. ed. Porto Alegre: Bookman, 2006. 496 p.

MINTZBERG, H.; AHLSTRAND, B.; LAMPEL, J. Safári de estratégia: um roteiro pela selva do planejamento estratégico. 2. ed. Porto Alegre: Bookmann, 2010. 392 p. Tradução de: Lene Belon Ribeiro.

MIZAEL, G. A. et al. Análise do plano de desenvolvimento institucional das universidades federais do consórcio sul-sudeste de Minas Gerais. Revista de Administração Pública, Rio de Janeiro, v. 47, n. 5, p.145-164, out. 2013.

OLIVEIRA, S. B.; TODA, F. A. O planejamento estratégico e a Visão Baseada em Recursos (RBV): uma avaliação da tecnologia da informação na gestão hospitalar. Revista Eletrônica de Ciência Administrativa, v. 12, n. 1, p. 39-57, 2013.

PAULI, J.; BIULCHI, A. F. Avaliação de desempenho no serviço público: a experiência do Instituto Nacional do Seguro Social na implantação da gratificação de avaliação de desempenho do seguro social - GDASS. Revista de Administração IMED, [s.1.], v. 2, n. 2, p.129-137, 30 ago. 2012.

PINSONNEAULT, A.; KRAEMER, K. Survey research methodology in management information systems: an assessment. Journal of Management Information

Systems, Califórnia, v. 10, n. 2, p.75-105, 1993. 
RIBEIRO, A. M.; CARMO, C. H. S. Planejamento estratégico e sustentabilidade: uma análise da relação entre o conteúdo das missões institucionais das companhias brasileiras de capital aberto e a sua presença no índice de sustentabilidade da Bovespa. Revista de Gestão Social e Ambiental, v. 9, n. 1, p. 19-35, 2015.

SANTOS, F. J. S. dos; SANO, H. Inovação no setor público: um olhar sobre os estudos brasileiros. Revista Interface, Natal, v. 13, n. 2, p.33-48, dez. 2016.

SANTOS, J. L. S. et al. Modelo de planejamento estratégico em uma universidade pública brasileira: desenvolvimento e implementação na Universidade Federal de Alagoas. In: colóquio internacional sobre gestão universitária na América do Sul, 9., 2009, Florianópolis. Anais... . Florianópolis: AEGES, 2009. p. 1 - 16.

SANTOS, M. C. dos. Gestão da sustentabilidade: uma avaliação de meios de hospedagem em unidades de conservação. 2018. 77 f. Dissertação (Mestrado) - Curso de Mestrado Acadêmico em Administração, Programa de Pós-graduação em Administração, Universidade Estadual do Ceará, Fortaleza, 2018.

SANTOS, R. W. D.; NASCIMENTO, V. M. B.; OLIVEIRA, M. C. R. Gestão Estratégica de Pessoas e a Importância do Alinhamento das Diretrizes Estratégicas: Um Estudo de Caso. Revista de Carreiras e Pessoas, v. 8, n. 3, p. 413-430, 2018.

SILVA, C. L. da; ASSIS, L. aplicabilidade do planejamento estratégico em hospitais universitários federais. Revista de Gestão em Sistemas de Saúde, Anápolis, v. 5, n. 2, p.3956, dez. 2016.

SILVA, E. G. B. da. O Planejamento Estratégico para Implantação da sustentabilidade na Copa do Mundo FIFA 2014 no Brasil. Turismo em Análise, São Paulo, v. 27, n. 2, p.341363, ago. 2016.

SILVA, F. A.; GONÇALVES, C. A. O processo de formulação e implementação de planejamento estratégico em instituições do setor público. Revista de Administração da UFSM, Santa Maria, v. 4, n. 3, p.458-476, dez. 2011.

SILVA, L. P. da et al. Planejamento estratégico participativo e seus efeitos no clima organizacional. PRACS: Revista Eletrônica de Humanidades do Curso de Ciências Sociais da UNIFAP, [s.1.], v. 10, n. 2, p.79-95, 14 dez. 2017. Universidade Federal do Amapá. http://dx.doi.org/10.18468/pracs.2017v10n2.p79-95.

SOUZA, S. C.; MARINHO, S. V. Planejamento estratégico baseado no balanced scorecard: um estudo de caso aplicado a uma pequena empresa de segurança. Revista Gestão \& Planejamento, v. 15, n. 2, p. 213-237, 2014.

THOMPSON JUNIOR, A. A.; STRICKLAND III, A. J. Planejamento estratégico: elaboração, implementação e execução. São Paulo: Pioneira, 2000. 
UNIFAP. Universidade Federal do Amapá, Pró-Reitoria de Gestão de Pessoas. Planejamento Estratégico da PROGEP. Macapá: UNIFAP, 2016. Disponível em:

http://www2.unifap.br/drh/. Acesso em: 25 maio. 2019.

UNIFAP. Universidade Federal do Amapá, Pró-Reitoria de Gestão de Pessoas. Resolução no 01, de 27 de março de 2013. Cria a Pró-Reitoria de Planejamento.

UNIFAP. Universidade Federal do Amapá, Pró-Reitoria de Planejamento. Relatório de Gestão da Fundação Universidade Federal do Amapá: 2019. Macapá: UNIFAP, 2019. 129 p. Disponível em: < http://www2.unifap.br/deplan/relatorios-de-gestao/>. Acesso em: 28 maio. 2019.

UNIFAP. Universidade Federal do Amapá. Consulta do quantitativo de técnicoadministrativos e docentes por unidade, 2019. Emitido pelo Sistema Integrado de Gestão de Recursos Humanos - SIGRH. Disponível em: https://sigrh.unifap.br. Acesso em: 16 mar. 2019.

UNIFAP. Universidade Federal do Amapá. Plano de Desenvolvimento Institucional: 20152019. Macapá: UNIFAP, 2015. 113 p. Disponível em: http://www2.unifap.br/pdi/ . Acesso em: 04 out. 2018.

WRIGHT, P.; KROLL, M. J.; PARNELL, J. Administração estratégica: conceitos. 2000. 